\title{
A Signal to Noise Optimization Algorithm for Speckle Interferometry Applications
}

\author{
J. Molimard ${ }^{1 a}$, R. Cordero ${ }^{2,3 b}$, A. Vautrin ${ }^{1 c}$ \\ 1LTDS, UMR CNRS/ECL/ENISE/ENSMSE 5513, Ecole des Mines de Saint-Etienne, \\ 158 cours Fauriel, 42023 Saint-Etienne cedex 02, France \\ 2Departamento de Física, Universidad de Santiago de Chile, \\ Casilla 307, Correo 2, Santiago, Chile \\ ${ }^{3}$ Universidad Técnica Federico Santa María, \\ Ave. España 1680, Valparaíso, Chile
}

amolimard@emse.fr, brcordero@ing.puc.cl, `vautrin@emse.fr

Keywords: speckle - interferometry - shearography - correlation - signal to noise ratio

\begin{abstract}
Optical Full Field Techniques (OFFT) are more and more utilized by mechanical laboratories. Among these methods, interferometry techniques (mainly composed of Speckle/Grating Interferometry or Speckle/Grating Shearography) are more difficult to use in a mechanical lab context, because of their sensitivity to external vibrations (except shearography), and because of the global lack of optical culture of mechanical engineers. Speckle-based methods are of great practical interest for the users, but their signal to noise ratio (SNR) is affected by the rigid body motion of the specimen. Here, the speckle decorrelation is minimized at local scale directly using the SNR. First, a shearography experiment is modeled to characterize the recorrelation procedure for a rigid body motion, a constant strain map and finally a high degree of localization. The mean noise level is found to be 6 times higher than a fully-correlated phase map for a 1 pixel speckle size. Last, a first application to a single-ply fabric composite lamina is shown. Resulting strain maps are of high quality with a very low spatial resolution (4 pixels). The local bending / global tension coupling effect is clearly put in evidence.
\end{abstract}

\section{Introduction}

Optical Full Field Techniques (OFFT) are more and more utilized by mechanical laboratories. Among these methods, the kinematics measurements are of great interest. They can be classified in two main groups: geometric methods and interferometric methods. The former is composed of Grid Method (or Moiré geometry) and Digital Image Correlation among others; the latter of grating and speckle interferometry or grating and speckle shearography. The first group is easier to develop and use, but its sensitivity is poor. In so far, it is particularly useful to large displacements applications (metal forming [1]) or to damage monitoring (crack propagation [2]). The second group is more difficult to use in a mechanical lab context, because of its sensitivity to external vibrations (except shearography), and because of the global lack of optical culture of mechanical engineers and scientists. Among these methods, because they need few surface preparing (at worst, a white diffusive paint), speckle-based methods are of great practical interest for the users. But, their signal to noise ratio (SNR) is poor compared to grating interferometry or shearography.

This signal to noise ratio has been investigated by many authors and a theoretical as well a practical approach shows that the SNR is mainly function of the speckle correlation [3]. If the speckles are fully correlated, the noise is minimum. Then, the noise level can be addressed in this ideal case [4]. But, when the speckles are decorrelated, the noise level is maximum, i.e. the signal has completely vanished. The main contribution to the speckle decorrelation is the deformation outside the field of view, in other words the rigid body motion. A first strategy consists in applying 
small-enough load step in order to keep the correlation from one step to the other [5]. In many cases, a simple global translation of the phase maps before subtraction is enough to recover the signal [6]. But if the speckle size is small, as recommended by many authors, and if the displacement field is disturbed, a simple global translation is not enough and a local translation has to be defined and used.

A first idea consists in combining the speckle interferometry and the so-called "speckle photography" technique [7]. This latter is an image correlation technique. The latest development of this approach was presented by Svanbro [8]. The correlation coefficient was written using complex intensity maps, i.e. including the phase maps. However, the method seems to present some drawbacks in case of high fringe densities. Moreover it requires the acquisition of an additional intensity map, which increases the complexity of the procedure For a simple out-of-plane interferometry set-up, this should be negligible, but a complete shearography in-plane and out-ofplane procedure [9] already needs 24 images at most for a 3 arms set-up, with a classical 4-steps temporal phase extraction, and 56 images for a 4 arms set-up, with the Windowed Fourier Transform algorithm proposed by Surrel [10]. Obviously, the global trend is to simplify this apparatus by adding some automated capabilities, and adding one more operation is unwanted for the seek of simplicity and time-saving. In addition, image correlation is not well adapted to very small speckles (i.e. close to the pixel size). We propose here a new procedure directly dealing with the phase maps and optimizing the SNR at the local level.

A shearography set-up is modeled, and the recorrelation principle tested on different cases (translation, constant strain, high density fringes). Mean maximum decorrelation error is quantified. Then, a first practical application shows some results in the case of a single-ply fabric laminate.

\section{Signal to noise optimization}

\section{Principle}

Neither the optical set-up and or phase extraction technique will be considered in this section. The discussion should be independent of either. The only requirement is that an initial phase map be subtracted from a final phase map, giving a low-frequency information, as it is generally in the case for mechanical strain/displacement fields. Consequently, this work should be well adapted to speckle shearography or speckle interferometry. The section is centered on the description of the SNR optimization procedure, as it is shown Fig. 1. First, the initial phase map $\Phi^{\text {ini }}$ is divided into small regions of interest (ROI), typically $32 \times 32$ pixels, centered around the co-ordinates $\{i, j\}$. In the final phase map $\Phi^{f i n}$, ROI of the same size, centered around $\{i+\delta i, j+\delta j\}$ are subtracted to the initial sub-map.

$$
\Delta \Phi_{\{i, j\}}(\delta i, \delta j)=\Phi_{\{i+\delta i, j+\delta j\}}^{f i n}-\Phi_{\{i, j\}}^{i n i}
$$

The off-set vector $\{\delta i, \delta j\}$ varies within a certain range, corresponding to the expected translation, usually $\{ \pm 5, \pm 5\}$ pixels. The signal to noise ratio for a given ROI position and a given offset vector can be calculated. As these are phase maps, a phase jump is possible, changing dramatically the map frequency signature. To eliminate this difficulty, the sine of the phase difference is considered. It is split in two components, using a simple FFT algorithm:

- the low-frequency one, $\sin \left(\Delta \Phi_{\{i, j\}}(\delta i, \delta j)\right)^{L F}$, considered as a possible mechanical signal,

- the high-frequency one, $\sin \left(\Delta \Phi_{\{i, j\}}(\delta i, \delta j)\right)^{H F}$, considered as noise.

The signal, denoted $S(\delta i, \delta j)^{L F}$, can be defined either by the dynamic range of the low-frequency component or by the mean value of the low frequency component. In some cases, these two data sets can be close to zero, and may be replaced by a constant. The noise is defined by the standard 
deviation of the high-frequency component. Then, it is possible to calculate a SNR for each off-set $\{\delta i, \delta j\}$.

$$
\operatorname{SNR}_{\{i, j\}}(\delta i, \delta j)=\frac{S(\delta i, \delta j)^{L F}}{S T D\left(\sin \left(\Delta \Phi_{\{i, j\}}(\delta i, \delta j)\right)^{H F}\right)}
$$

The frequency filter has to be adjusted arbitrarily. In the following, it will be taken to $1 / 5^{\text {th }}$ of the sub-map size. A SNR map can be drawn considering any combination of the off-set vector. The optimum translation corresponds to the vector giving the highest SNR.

Last, entire displacement maps according to $\mathrm{x}$ and $\mathrm{y}$ directions are calculated. These maps can be processed as any other displacement maps. In particular, even in optimal conditions these maps are of good quality, a median filter can be useful to remove incoherent points. Then, the local displacement maps are taken into account in the phase subtraction procedure to calculate the optimum global phase difference map. No sub-pixel capability is addressed yet. This should be done in the future if necessary, but it needs some precautions in the interpolation of speckle phases, because of the randomness characteristic of a speckle chart. In particular, the lack of continuity between phase speckles prohibits any interpolation algorithm, either in direct or Fourier space. Furthermore, it would increase the time processing.

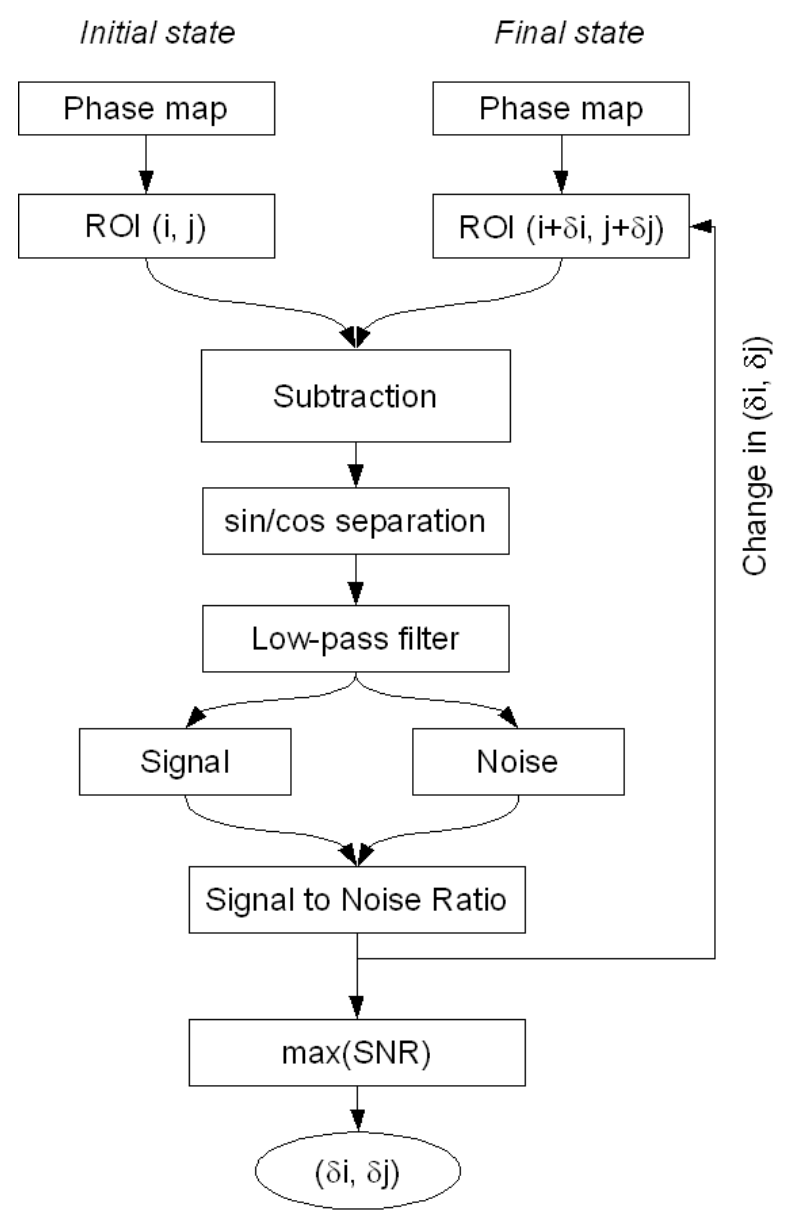

Figure 1. Flow chart of recorrelation procedure.

As in image correlation techniques, many parameters require fine tune adjustment: the ROI size, the off-set range, the displacement grid step, the low/high frequency threshold... Optimization of these parameters should be addressed carefully in the future. In the mean time, some simple 
considerations can be used: note that the ROI should be adapted to the expected gradient within the field in order to have sufficiently localized information. But if the ROI is too small, the signal / noise discrimination, i.e. the extraction of the mechanical information becomes impossible. Here, the ROI size is set to $32 \times 32$ pixels $^{2}$, as it is commonly used in classical digital image correlation techniques. Note that no assumption is made in the nature of the displacement (strain) fields . Usually, these fields are continuous, but discontinuities could occur (cracks, bolt joining, contacts ...). The knowledge of the user should be introduced as a tool to verify the quality of the fields, and an incoherent displacement should be removed. Here, the choice of no prior knowledge on the displacement (strain) field is justified by the generality of the approach. Last, the procedure does not consider the possibility of a rigid body rotation. This should dramatically decrease the SNR ratio. This point would have to be addressed if necessary.

\section{Simulated field}

\section{Speckle simulation principle}

The speckle simulation used here is based on previous work by Equis [11]. A randomly distributed complex amplitude field is generated. Then, the speckle size is adjusted using a Gaussian low-pass filter. Because we aim to apply this approach to speckle shearography, the amplitudes are combined as it would be in a classical Michelson interferometer. If the shearing distance is lower than the speckle size, the initial and final phase map should be correlated, then results should be different than speckle interferometry results. Last, phases are averaged over a certain area, simulating the pixel recording.

A simulated mechanical loading is applied to the field. It acts in two directions: first, points are moved, leading to the basic decorrelation process. Second, the phase is changed according to the sensitivity vector used. We consider here a single source under a shearography set-up, with a sensitivity given by:

$$
\Delta \varphi(i, j)=\frac{4 \pi}{\lambda} \cdot \sin (\theta) \cdot \delta_{i} \cdot \frac{\partial u}{\partial x}+\frac{4 \pi}{\lambda} \cdot(1+\cos (\theta)) \cdot \delta_{i} \cdot \frac{\partial w}{\partial x}
$$

where $\lambda$ is the illumination wavelength, $\theta$ is the angle between illumination and observation directions, $\delta_{i}$ is the shearing distance, $\partial u / \partial x$ and $\partial w / \partial x$ being the kinematics changes of the surface, respectively one in-plane strain, and one out-of-plane rotation.

Now, the phase map between the two latter situations can be easily calculated as the angle of the difference of the two complex amplitudes.

\section{Simulated loading procedure}

Simulated conditions are chosen to be close to common practice in laboratories [9]. Speckle size is a priori close to the pixel size, thus its standard deviation is chosen to be 1 pixel. Even if in common practice, the shearing distance is usually higher, we use here a small value of 3 pixels, close to the most recent developments in our laboratory. In this particular case, speckles are fully decorrelated in the Michelson interferometer. The pixel size is set to $40 \mu \mathrm{m}$. Last, illumination source is assumed to be a doubled YAG Laser, so the wavelengh $\lambda=532 \mathrm{~nm}$, and the illumination direction $\theta=\pi / 4$. Last, a random phase noise of $1 / 100$ fringe is added to the signal. The covered field of view is 500 pixels $(20 \mathrm{~mm})$.

\section{Rigid body translation}

A simple translation is applied to the speckle map. It can be easily verified that standard deviation on the phase difference increases as a function of the translation, and that a complete 
decorrelation is achieved when the translation is 3 times the standard deviation of the speckle (blue cross, Fig. 2). Re-correlation procedure in applied for each case. Due to the specific nature of the test (the signal is uniformly equal to zero), the inverse of the standard deviation is taken as a signature of the signal to noise ratio.

Results show the efficiency of the re-correlation procedure (red circles, Fig. 2). Maximum errors are obtained for translations of $(n+0.5)$ pixels. The maximum error value corresponds to the 0.5 pixel translation without recorrelation technique. Considering an equally probable occurrence of each translations in a given map, the mean error is $0.39 \mathrm{rad}$ i.e. 6/100 fringe. Another test was performed with a larger speckle size. The trends remained the same, but the maximum is decreased corresponding again to the $(n+0.5)$ pixel translation. For a speckle 3 times larger, the mean noise level is 2.2 times smaller.

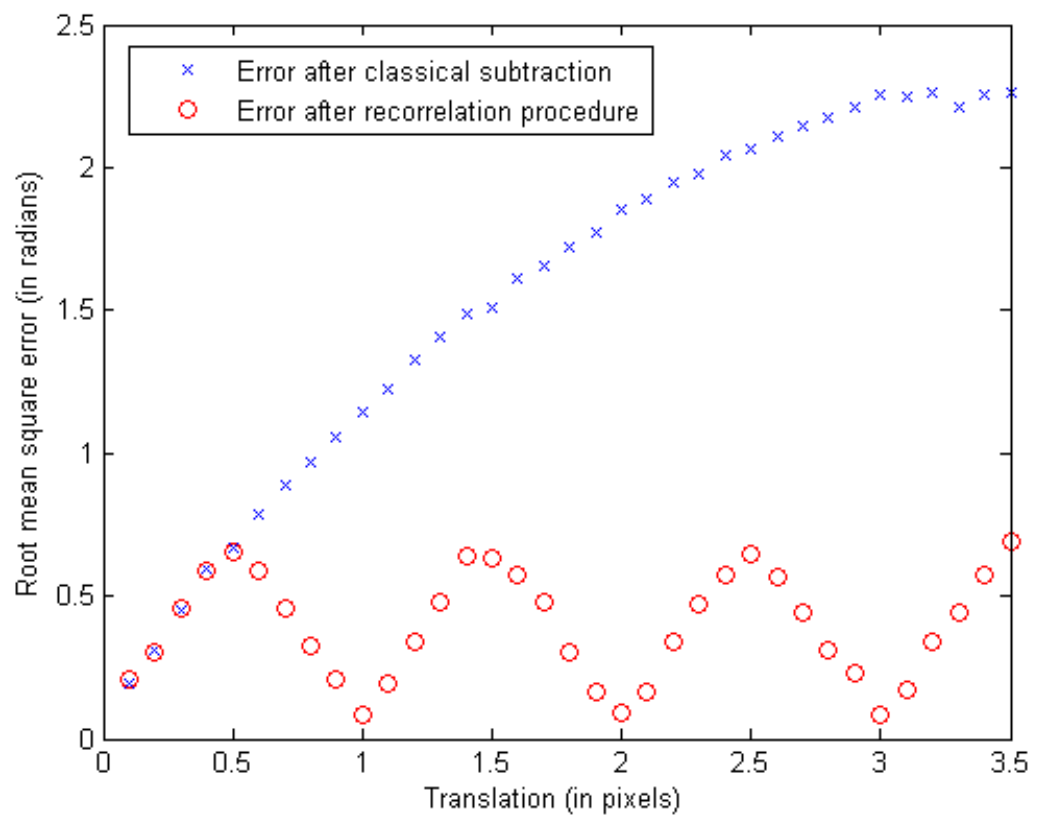

Figure 2. Decorrelation as a function of the translation between 2 steps.

\section{$1^{\text {st }}$ and $2^{\text {nd }}$ order displacement gradient}

A simple tensile test with data corresponding to the previous case is performed with a constant $5.10^{-3} \mu \mathrm{m} / \mathrm{m}$ longitudinal strain. On the left boundary, the displacement is null, and increases linearly up to 3 pixels $(120 \mu \mathrm{m})$ on the right boundary. Results are shown in Fig. 3. The interest of such recorrelation technique is outlined Fig. 3a and 3b: with a simple classical subtraction, complete decorrelation is achieved on the right side. In this case of a global translation of -1 pixel, partial decorrelation takes place on both sides, with a maximum noise level of $18.8 / 100^{\mathrm{e}}$ fringe on the left boundary and $29.2 / 100^{\mathrm{e}}$ fringe on the right boundary. In fact, it is impossible to find a global translation to minimize the noise at all points, hence a local procedure is necessary. Fig. 3c shows that most of the estimated displacements are reasonable. Apparent noise varies within the map as described before and is minimum for a $n$-pixels displacement. Some salt and pepper noise remains for $(n+0.5)$ displacement. A simple post-treatment could find and remove these points. Here, no post-treatment is applied in order to point out the effect of the correlation procedure alone.

Last, it could be important to test the efficiency of the method in case of a strong strain gradient, i.e. with a high fringe density. A sine-wave strain is simulated in order to reach large enough displacements to have decorrelation and sufficient strain gradient to have 5 pixels/fringe for a correlation window of 32 pixels. Again, the efficiency of the recorrelation procedure is evident (Fig. 4). 


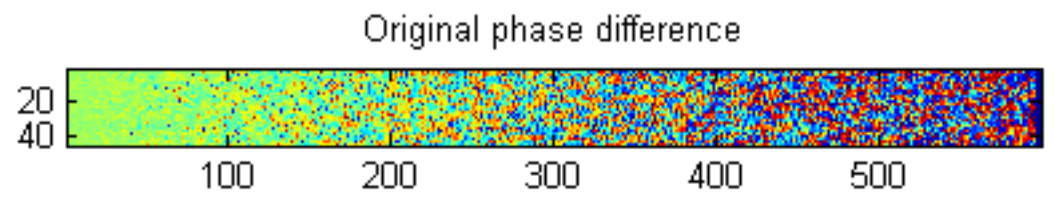

a)

Global translation of 1 pixel

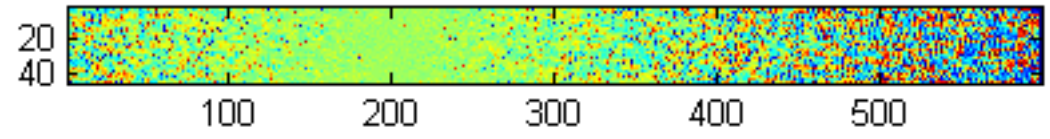

b)

After recorrelation procedure

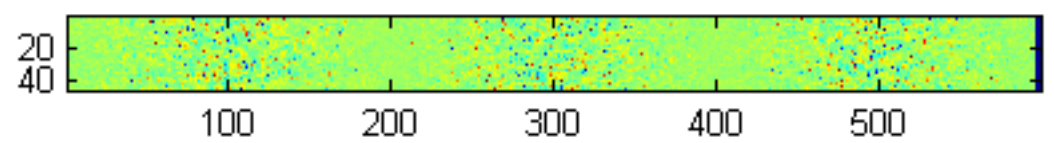

c)

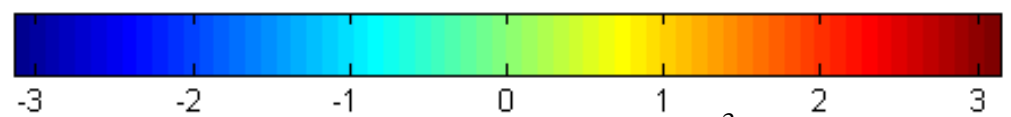

Figure 3. Original and recorrelated phase difference for a $3.10^{3} \mu \mathrm{m} / \mathrm{m}$ homogeneous strain (in rad).

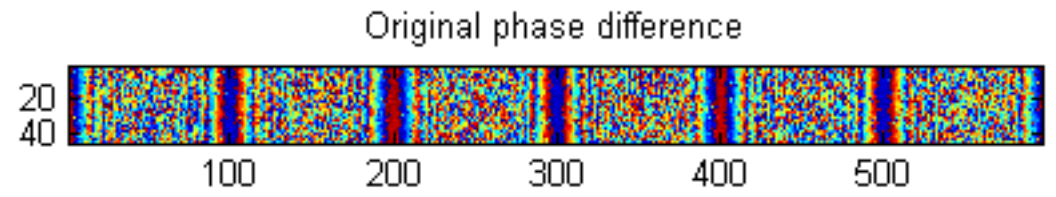

a)

After recorrelation procedure

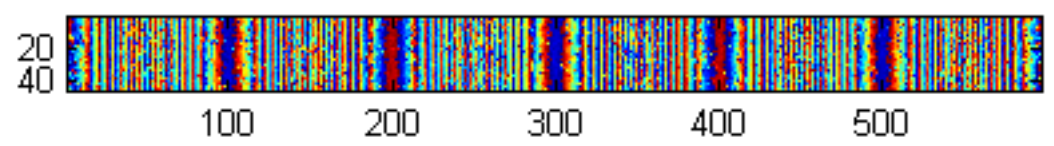

b)

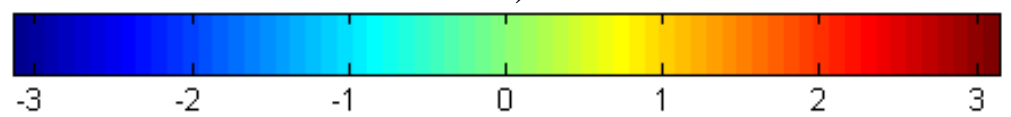

Figure 4. Original and recorrelated phase difference for a sine-wave strain field (in rad). 


\section{Application to a single-ply fabric laminate}

\section{Optical set-up}

Phase maps have been recorded using a 4-beam portable shearography set-up. It is composed of an $110 \mathrm{~mW}$ Diode-Pumped Solid-State (DPSS) laser with a wavelength of $532 \mathrm{~nm}, 5$ optical fibres, a Michelson interferometer, a CCD camera and a PC. The principles of this method based on different illumination beams to obtain the displacements derivatives have been already presented by Lee [12] or Bounda [9].

The set-up presented here uses four illumination beams, two symmetrical in the horizontal plane and two symmetrical in the vertical plane. The experimental procedure consists in recording 8 phase maps (4 illumination beams, and 2 shearing directions) before and after loading. In such a configuration, ESPSI allows a direct measurement of the displacement derivatives maps on the surface, and consequently strains and out-of-plane rotations. The sensitivity can be adjusted by tuning the shear distance $\delta_{i}$, avoiding the fringe collapse due to strain concentration. In the following experiments, the system parameters are an illumination angle $\theta=\pi / 4$ for all the illumination beams and the shearing distance $\delta_{i}=200 \mu \mathrm{m}$ for $\mathrm{x}$ and y directions. These last values are obtained by using the grid method [13].

\section{Specimen}

The specimen is a T700S/M10 12K plain-weave carbon fabric (48192, Hexcel Corporation), having the waviness $\left(\mathrm{h}_{\mathrm{y}} / 2 \mathrm{a}\right)$ of 0.0078 , as already used by Lee [12]. The single ply composite is cured using the procedure recommended by the supplier. The size of one unit cell is about $8 \times 8$ $\mathrm{mm}^{2}$ and the inspecting zone contains six unit cells.

\section{First results}

Phase maps are recorded at $50 \mathrm{~N}$ increments, from 200 to $450 \mathrm{~N}$. Here, we only focus on the tensile direction maps, either the $-\pi / 4$, or $+\pi / 4$ illumination angle. The difference of the two is proportional to the strain $\varepsilon_{x x}$, and the sum to the surface out-of-plane slope $\partial w / \partial x$. If we consider the $200 \mathrm{~N}$ load case and the $300 \mathrm{~N}$ load case, the difference shows some local decorrelation (Fig. 5a). Then, the proposed recorrelation procedure is applied (Fig. 5b). Some quality assessments were necessary: erroneous displacements can be easily isolated by comparing the displacement maps corresponding to each of the two phase maps. Then, displacement values are removed and replaced by an interpolated value. Last, salt and pepper noise were partially removed using a statisticallybased procedure detecting the $10 \%$ least probable points. 


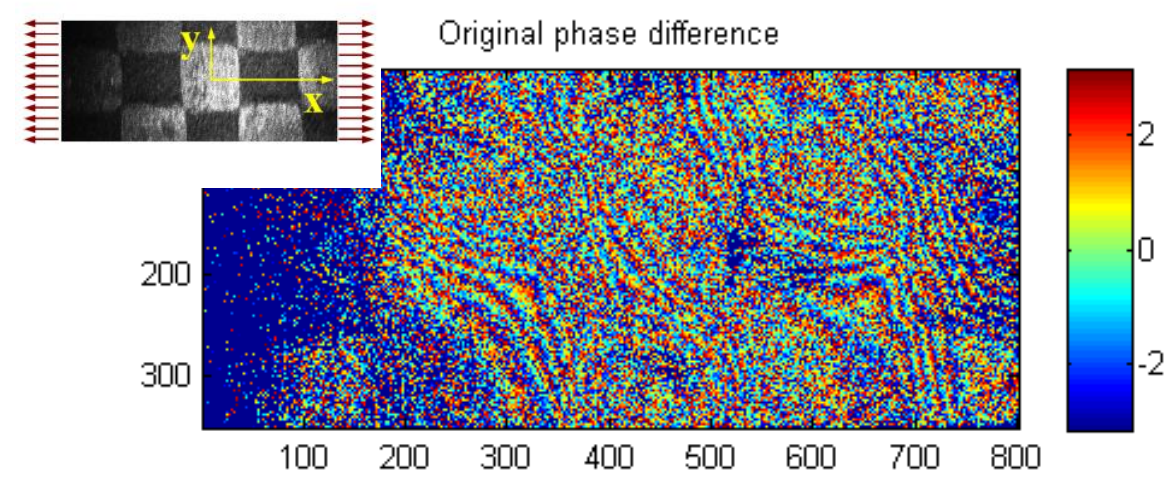

a)

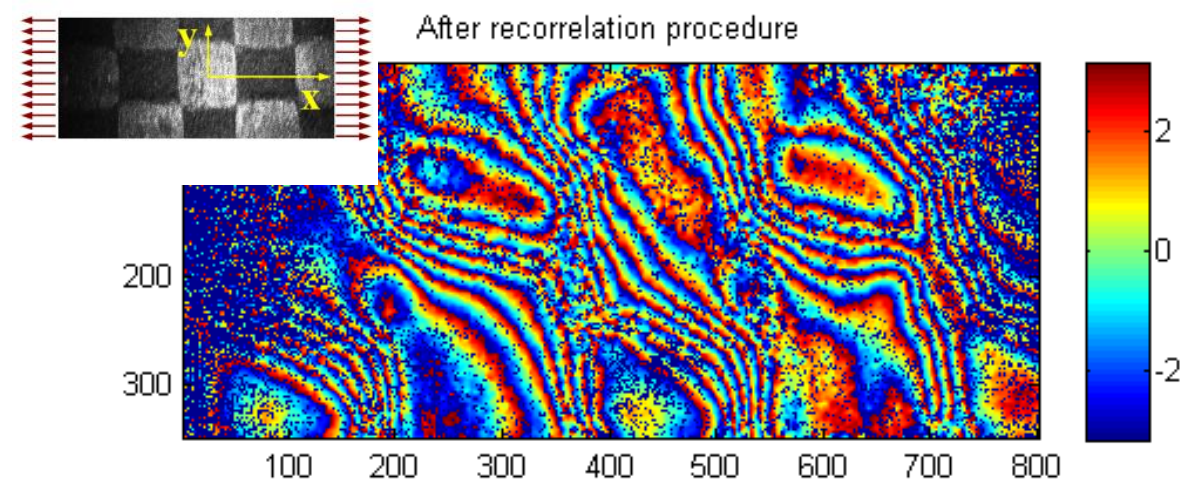

b)

Figure 5. Real experimental map on a fabric specimen phase difference without (a) and with recorrelation procedure (b).

\section{Mechanical interpretation}

Finally, it is possible to calculate the strain component $\varepsilon_{x x}$ and the slope $\partial w / \partial x$. Fig. 6 shows raw results, the spatial resolution remains 4 pixels. Any classical post-processing should be applied to decrease the apparent noise. In this particular case, speckle size is lower than 1 pixel, leading to a rather bad situation. From a mechanical perspective, results are quite classical, and correspond to previous results from Lee [12]: the weaves transverse to the loading direction are strongly deformed, and the longitudinal ones show a smaller strain (the fabric structure is represented schematically by the yellow lines). This effect can be explained by the coupling between global tension and local flexure effect. Here in particular, the shape indicates a misalignment between the longitudinal weaves and the loading direction. A better inspection of the specimen reveals that it contains some waviness in the plane of the specimen itself, probably due to the manipulation of the prepreg. 


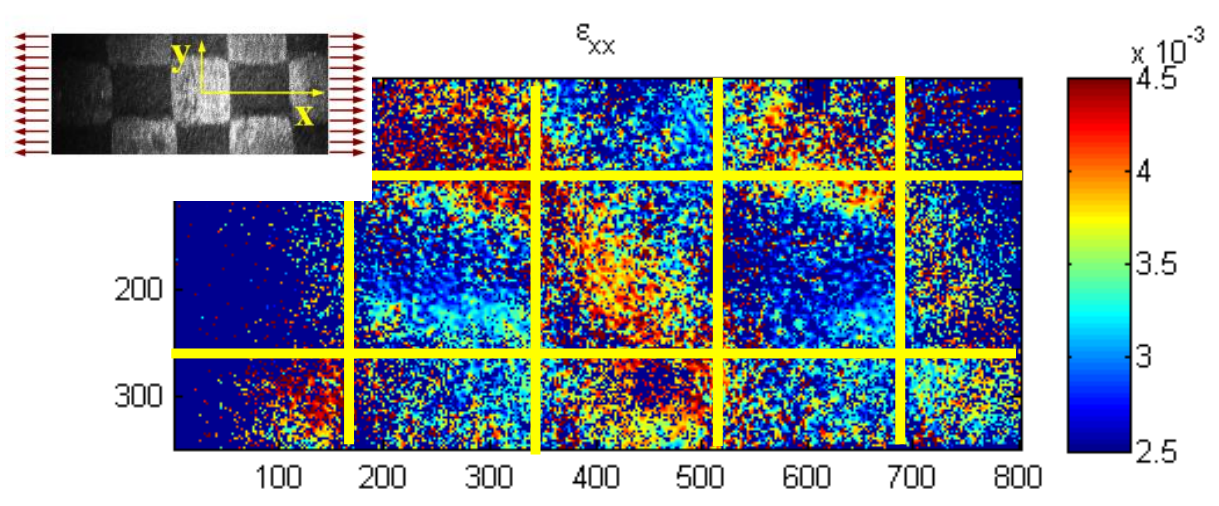

a)

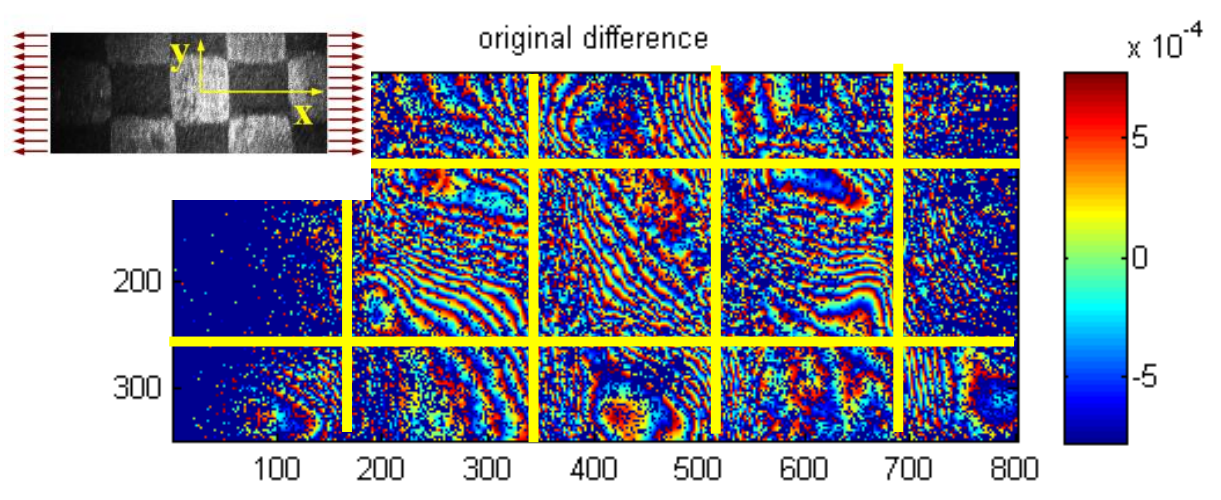

b)

Figure 6. Strain (a) and wrapped slope (b) for a $100 \mathrm{~N}$ load step.

\section{Conclusion}

The weak point of speckle techniques is their spatially random wavefront, and consequently a possible decorrelation of the speckles. In a classical mechanical context, rigid body motion is a very common problem. Then, it is absolutely necessary to address this point when using a speckle-based technique. Some solutions exist already, such as adding intermediate states or speckle correlation, but these solutions don't focus on the basic problem, which is the optimization of signal to noise ratio. In this article, we developed a simple way maximizing this data locally. A simulation was used to study some basic properties. Error level due to remaining decorrelation is completely known, and an error propagation model can be used on good basis. Last, a first example on a real experimental map is presented. Results show good trends, even without the use of an additional low-pass filter, except for a salt and pepper filter.

\section{Future work}

This paper presented a first attempt at localized optimization of signal to noise ratio in phase difference methods. Consequently, there are still many points to be developed. In particular, the use of multiple internal parameters (filter cut-off, ROI size) is an important drawback of the method, and should be clarified in the future. More theoretical points should be addressed to further decrease the noise, especially in case of low speckle size: first, some mechanical considerations such as displacement continuity should permit to avoid aberrant points, and give a better sub-pixel estimation of displacement maps. Then, noise sub-pixel interpolation of speckle phase will have to be developed to better re-correlate the phase maps. 
The authors would like to thank Y. Zhou, Msc, and Dr. C. Fournier from Hubert Curien Laboratory of the University of Saint-Etienne for their collaboration to this work.

\section{Bibliography}

[1] Brunet M., Touchal S., Morestin F., Numerical and experimental analysis of necking in 3D. Sheet forming prosesses using damage variable, Advanced Methods in Materials Processing Defects, M. Predeleanu \& P.Gilormini, Elsevier Science BV (1997) p. 205 -214.

[2] Rethore J., Roux S., Hild F., Noise-robust stress intensity factor determination from kinematic field measurements. Engineering Fracture Mechanics. Available online 3 May 2007 (Science Direct).

[3] Bruno L., Pagnotta L., Poggialini A., Laser speckle decorrelation in NDT, Optics and Lasers in Engineering, 34 (2000) p. 55-65.

[4] Cordero R.R., Molimard J., Martinez A., Labbe F., Uncertainty analysis of temporal phasestepping algorithms for interferometry, Optics Communications, 275 (2007) p. 144-155.

[5] Davila A., Huntley J.M., Kaufmann G. H., Kerr D., High-speed dynamic speckle interferometry: phase errors due to intensity, velocity, and speckle decorrelation, Appl Opt., 44, 19 (2005) p. 39543962

[6] Hung Y.Y., Wang J.Q., Hovanesian J. D., Technique for Compensating Excessive Rigid Body Motion in Nondestructive Testing of Large Structures Using Shearography, Optics and Lasers in Engineering, 26 (1997) p. 249-250.

[7] Martınez-Celorio R.A., Barrientos B., Sanchez-Marın F.J., Lopez L.M., Rayas J.A., Out-ofplane displacement measurement by electronic speckle pattern interferometry in presence of large in-plane displacement, Optics Communications, 208 (2002) p. 17-24.

[8] Svanbro A, DSPI: complex amplitude correlation for large in-plane compensations and phase evaluation, Proceedings of Speckle'06, SPIE vol. 6341 (2006) p. 63410I-1 5..

[9] Molimard J., Bounda D., Vautrin A., Quantitative strain and slope evaluation on a double lap joint tensile test using ESPSI, Proceedings of Speckle'06, SPIE vol. 6341 (2006) p. 63412R-1 6.

[10] Surrel Y., Design of algorithms for phase measurements by the use of phase stepping, Applied Optics 35, 1 (1996) p. 51-60.

[11] Equis S., Jacquot P., Simulation of Speckle Complex Amplitude : Advocating the Linear Model, Proceedings of Speckle'06, SPIE vol. 6341 (2006) p. 634136-1 6.

[12] Lee J.R., Molimard J., Vautrin A., Surrel Y., Digital phase-shifting grating shearography for experimental analysis of fabric composites under tension, Composites: Part A, 35 (2004) p. 849859.

[13] Surrel Y., Moiré and grid methods in optics, SPIE 2342, pp. 213-220 (1994). 